\title{
Effects of Nutrition on Osteoarthritis in Women Over the 50 Years: Using the Korea National Health and Nutrition Examination Survey (KNHANES) Data
}

\author{
Kyeong-Rae Kim ${ }^{1}$, Jae-Eun Park ${ }^{1}$, So-Young Lim ${ }^{1}$, Ho Kim ${ }^{2}$, Il-Tae Jang ${ }^{3} \&$ Kwang-Yeol Lee ${ }^{4}$ \\ ${ }^{1}$ Nanoori Medical Research Institute, Nanoori Hospital Gangnam, Seoul, Republic of Korea \\ ${ }^{2}$ 2bko, Seoul, Republic of Korea \\ ${ }^{3}$ Department of Neurosurgery, Nanoori Hospital Gangnam, Seoul, Republic of Korea \\ ${ }^{4}$ Department of Orthopedics, Nanoori Hospital Gangnam, Seoul, Republic of Korea \\ Correspondence: Kwang-Yeol Lee, Department of Orthopedics, Nanoori Hospital Gangnam, Eonju-ro, \\ Gangnam-gu, Seoul, Republic of Korea. Tel: 82-2-6003-9767. E-mail: osman10@naver.com
}

Received: March 24, 2020 Accepted: April 17, 2020 Online Published: May 8, 2020

doi:10.5539/gjhs.v12n7p29 URL: https://doi.org/10.5539/gjhs.v12n7p29

\begin{abstract}
There are prior articles on osteoarthritis of demographic and nutritional factors, previous studies show a lack of empirical analysis using public data, focusing primarily on predicting or assessing risk factors focusing on demographic characteristics. Since the disease called osteoarthritis itself has yet to be clearly treated, we would like to establish that the preventive medical aspect of osteoarthritis is very important and that prevention through nutrient intake can reduce the social and economic costs of osteoarthritis. We intend to prepare basic data for osteoarthritis by analyzing the prevalence of osteoarthritis in women over the 50 age based on demographic and nutritional characteristics using the 7th National Nutrition Survey data in 2016 and 2017. As results, cholesterol and sodium negatively affect the odds of osteoarthritis and nutrients classified as inorganic reduce the odds of osteoarthritis in 50 age women to 59 years old. In addition, in women over 60 and under 69 years of age, vitamin B has been found to reduce the risk of osteoarthritis and iron has a significant effect on women in their 70s and older. Therefore, these nutrients are 'micronutrients' and, among the five major nutrients, were identified as the nutrients that assisted the 'macronutrients'.
\end{abstract}

Keywords: cross-sectional studies, logistic models, osteoarthritis

\section{Introduction}

Osteoarthritis is a degenerative disorder that damages the cartilage, bones and ligaments, and other essential components of joints which leads to inflammation and pain. In the past, osteoarthritis was called 'degenerative arthritis' and was known as a condition mainly related to aging (Ha et al., 2019). Presently, osteoarthritis is a disorder known to have multiple causes and not only age-related. Severity over time and symptoms emerge and change for individual patients depending on complex factors including age, genetic predisposition, obesity, joint shape, and hormones (CDCP., 2019; Fauci et al., 2008).

In South Korea, the prevalence of osteoarthritis in the age groups 44 years old or older is 201.2 per 1000 for men, and 472.6 per 1000 for women, which is a high-level of morbidity, and accordingly, there are a growing number of people who suffer from pain and physical impairments (Hur, 2008). Also, the prevalence of osteoarthritis tends to rise gradually as age increases, therefore is reported as one of the leading factors contributing to joint disorders in middle-aged and older people but not among younger people (Breedveld, 2004).

Although risk factors for osteoarthritis have not been comprehensively identified, it seems from previous studies that this disorder is mainly due to interactions among genetic and environmental factors. An epidemiological study showed that 39-65\% of osteoarthritis cases are caused by genetic factors (Spector, 1996), and other studies report that men, higher than the average body-mass index (BMI), and being in an older age group are major risk factors for osteoarthritis(Pearson-Ceol, 2007; Zakkak, 2009). In Korea, the prevalence of musculoskeletal disorders and other various chronic diseases has been on the rise as median age and life expectancy increase, and the reported data show that the prevalence of osteoarthritis is increasing in women, not men (Cho, 2011). 
There have been active attempts to identify the effect of nutrition on osteoarthritis. Calcium, antioxidants, vitamins (in particular B vitamins), and omega-3 fatty acids have been reported to be helpful for arthritis (AMC, 2019), and recent findings have reported that cholesterol causes degenerative arthritis if it excessively accumulates in articular cartilage(Choi, 2019).

While earlier data suggest demographic and nutritional factors contributing to osteoarthritis, these previous studies focused on predicting or characterizing risk factors based mostly on demographic characteristics (Kim et al., 2011; Gardiner et al., 2015), and therefore, it seems that few empirical studies have been conducted using publicly available data. Since there is no method, other than surgery, to treat osteoarthritis effectively, it is critical to prevent osteoarthritis and prevention through proper nutrition could reduce the socioeconomic costs of osteoarthritis.

Thus, the present study's goal is to provide a primary reference for preventing osteoarthritis in women 50 years old and older by analyzing the prevalence of osteoarthritis in this population and considering their demographic and nutritional features using data from the 7th Korea National Health and Nutrition Examination Survey 2016-2017 (KNHANES).

\section{Materials and Methods}

\subsection{Study Design}

The present study used the data from KNHANES, which consists of four parts: (1) Health Interview Survey - that examines disease morbidity, limited activity, accident injury, and healthcare utilization; (2) Health Behavior Survey - which relates to drinking and smoking; (3) Health Examination Survey; and (4) Nutrition Survey. The present study was conducted pursuant to the Ethical Principles for Medical Research Involving Human Subjects under the Declaration of Helsinki.

The participants were stratified by government administrative district-type (e.g., districts such as 'dong,' 'eup,' or 'myeon'), residential type (apartment or detached house) and regionally by seven Metropolitan Cities and six provinces including Gyeonggi-do, Gangwon-do, Chungcheong-do, Jeolla-do, Gyeongsang-do, and Jeju-do Island) based on the Population and Housing Census. Participants were allocated in proportion to each stratum's population for each district-type and then selected with systematic sampling. Then all members of the selected households were surveyed. The present study used survey data for women 50 years old or older from this sample. Sample data included information about participants' age, education, income, physical activity, tobacco use, alcohol consumption, osteoarthritis history, and nutrient consumption. Of the 17,658 total persons in the KNHANES data, all men, women less than 50 years old, and women with chronic diseases or key variables missing were excluded. Thus, 2,084 participants remained and were included in this study. The 7th KNHANES obtained its exemption from the Institutional Review Board of the Korea Centers for Disease Control and Prevention.

\subsection{Study Variables}

The dependent variable used in the present study was the presence or absence of osteoarthritis. The control variables were set as income level, education level, place of residence, physical activity, type of occupation, marital status, private health insurance, economic activity, drinking, and smoking. The independent variables used in the model included proteins, fats, saturated fatty-acids, monounsaturated fatty-acids, polyunsaturated fatty-acids, n-3 fatty-acids, n-6 fatty-acids, cholesterol, carbohydrate, dietary fiber, calcium, phosphorus, iron, sodium, potassium, vitamin A, carotene, retinol, thiamine, riboflavin, and niacin. All the independent variables were measured as the amount of intake right before the survey date. The dependent variable osteoarthritis was surveyed based on arthritis history as diagnosed by the physician.

\subsection{Analysis Method}

The present study calculated osteoarthritis prevalence depending on demographic variables and performed the Cochran-Mantel-Haenszel test to examine the significance of grouping and categorical variables. The independent $\mathrm{t}$-test was conducted for continuous variables. Osteoarthritis prevalence adjusting for all covariates was designated as either 'No' (no osteoarthritis) or 'Yes' (osteoarthritis) for each item, and then logistic regression was performed. Multi-variable logistic regression adjusting for confounding variables was performed for the odds ratio (OR) and with a 95\% confidence level for the confidence interval (CI) of osteoarthritis prevalence in each group by age, and all statistical analyses were analyzed using STATA/SE 13.0 version.

\section{Results}

\subsection{Sample Characteristics}

Table 1 shows the differences in demographic characteristics between the osteoarthritis group and the 
non-osteoarthritis group.

Table 1. Osteoarthritis prevalence depending on demographic characteristics

\begin{tabular}{|c|c|c|c|c|c|}
\hline \multirow{3}{*}{ Variable } & \multicolumn{4}{|c|}{ Osteoarthritis } & \multirow{3}{*}{$\mathrm{p}$} \\
\hline & \multicolumn{2}{|l|}{ No } & \multicolumn{2}{|l|}{ Yes } & \\
\hline & $\mathrm{N}$ & $\%$ & $\mathrm{~N}$ & $\%$ & \\
\hline & 1598 & 69.9 & 689 & 30.1 & \\
\hline Income & 1473 & & 611 & & \multirow{6}{*}{$<0.01$} \\
\hline Low & 254 & 17.2 & 135 & 22.1 & \\
\hline Low-moderate & 275 & 18.7 & 128 & 20.9 & \\
\hline Moderate & 283 & 19.2 & 114 & 18.7 & \\
\hline Moderate-high & 336 & 22.8 & 107 & 17.5 & \\
\hline High & 325 & 22.1 & 127 & 20.8 & \\
\hline Place of residence & 1604 & & 692 & & \multirow{3}{*}{0.688} \\
\hline Rural & 281 & 19.1 & 110 & 18.0 & \\
\hline Urban & 1192 & 80.9 & 501 & 82.0 & \\
\hline Age & 1604 & & 692 & & \multirow{4}{*}{$<0.01$} \\
\hline $50-59$ & 621 & 42.2 & 109 & 17.8 & \\
\hline $60-69$ & 469 & 31.8 & 214 & 35.0 & \\
\hline Over 70 & 383 & 26.0 & 288 & 47.1 & \\
\hline Education level & 1591 & & 682 & & \multirow{5}{*}{$<0.01$} \\
\hline$\leq 6$ years & 567 & 38.5 & 345 & 56.5 & \\
\hline $7-9$ years & 255 & 17.3 & 124 & 20.3 & \\
\hline $10-12$ years & 387 & 26.3 & 94 & 15.4 & \\
\hline$\geq 13$ years & 264 & 17.9 & 48 & 7.9 & \\
\hline Type of occupation & 1593 & & 682 & & \multirow{6}{*}{$<0.01$} \\
\hline Unemployed & 158 & 10.7 & 14 & 2.3 & \\
\hline Office work & 52 & 3.5 & 8 & 1.3 & \\
\hline Sales and Services & 218 & 14.8 & 63 & 10.3 & \\
\hline Agriculture, forestry and fishery & 287 & 19.5 & 125 & 20.5 & \\
\hline Machine fitting and simple labor & 758 & 51.5 & 401 & 65.6 & \\
\hline Marital status & 1604 & & 692 & & \multirow{4}{*}{0.464} \\
\hline Married & 1360 & 92.3 & 562 & 92.0 & \\
\hline Single & 98 & 6.7 & 45 & 7.4 & \\
\hline bereavement & 15 & 1.0 & 4 & 0.7 & \\
\hline Private health insurance & 1598 & & 688 & & \multirow{3}{*}{$<0.01$} \\
\hline No & 457 & 31.0 & 277 & 45.3 & \\
\hline Yes & 1016 & 69.0 & 334 & 54.7 & \\
\hline Economic activity & 1595 & & 682 & & \multirow{3}{*}{$<0.01$} \\
\hline No & 837 & 56.8 & 409 & 66.9 & \\
\hline Yes & 636 & 43.2 & 202 & 33.1 & \\
\hline
\end{tabular}




\begin{tabular}{llllll}
\hline Smoking & 1593 & & 683 & \\
Non-smoker and Ex-smoker & 1375 & 93.3 & 575 & 94.1 & 0.793 \\
Current Smoker & 98 & 6.7 & 36 & 5.9 & \\
\hline Drinking & 1593 & & 685 & & $<0.01$ \\
2 drinks/month to 3drink/week & 1096 & 74.5 & 407 & 66.6 & 33.4 \\
4 drinks/week & 376 & 25.5 & 204 & \\
\hline
\end{tabular}

Using income data by quintile, differences across each income quintile were evaluated between the No-group and the Yes-group $\left(\chi^{\wedge} 2=12.70, p<0.001\right)$. For the place of residence, no difference was observed between the two groups $\left(\chi^{\wedge} 2=0.326, p=0.688\right)$. In age, there was a difference between the two groups $\left(\chi^{\wedge} 2=134.16, p<0.001\right)$. In the Yes-group, participants tended to be diagnosed with osteoarthritis more as age increased. A difference between the two groups was also found for education level $\left(\chi^{\wedge} 2=85.4, \mathrm{p}<0.001\right)$. There was also a difference between the two groups for occupation $\left(\chi^{\wedge} 2=66.88, \mathrm{p}<0.001\right)$. No difference was found between the two groups in terms of marital status $\left(\chi^{\wedge} 2=0.951, \mathrm{p}=0.464\right)$. Private health insurance $\left(\chi^{\wedge} 2=38.76, \mathrm{p}<0.001\right)$ and economic activity $\left(\chi^{\wedge} 2=18.38\right.$, $\mathrm{p}=0.001$ ) showed a difference between the groups. For smoking and alcohol consumption, a difference between the two groups was confirmed only for alcohol consumption $\left(\chi^{\wedge} 2=13.22, \mathrm{p}<0.001\right)$.

\subsection{Nutrient Intake Differences Between Groups}

The study analyzed differences in nutrient intake between the two groups. Variables used in this analysis are continuous. Thus, an independent t-test was conducted (Table 2).

Table 2. Osteoarthritis prevalence depending on nutrient intake

\begin{tabular}{|c|c|c|c|c|c|}
\hline \multirow{3}{*}{ Variable } & \multicolumn{4}{|c|}{ Osteoarthritis } & \multirow{3}{*}{$\mathrm{p}$} \\
\hline & \multicolumn{2}{|l|}{ No } & \multicolumn{2}{|l|}{ Yes } & \\
\hline & Mean & Std.dev & Mean & Std.dev & \\
\hline proteins & 54.25 & 26.39 & 48.66 & 26.49 & $<0.01$ \\
\hline fats & 30.36 & 22.74 & 25.72 & 20.90 & $<0.01$ \\
\hline saturated fatty-acids & 8.54 & 7.23 & 7.24 & 6.38 & $<0.01$ \\
\hline monounsaturated fatty-acids & 9.30 & 8.06 & 7.70 & 7.07 & $<0.01$ \\
\hline polyunsaturated fatty-acids & 8.34 & 6.98 & 7.08 & 6.52 & $<0.01$ \\
\hline n-3 fatty-acids & 1.55 & 1.90 & 1.36 & 1.73 & .033 \\
\hline n-6 fatty-acids & 6.78 & 5.88 & 5.73 & 5.56 & $<0.01$ \\
\hline cholesterol & 180.63 & 199.77 & 136.60 & 178.91 & $<0.01$ \\
\hline carbohydrate & 276.20 & 115.47 & 261.98 & 110.86 & .010 \\
\hline dietary fiber & 24.35 & 14.58 & 22.98 & 14.22 & .049 \\
\hline calcium & 436.69 & 262.99 & 401.28 & 295.55 & .007 \\
\hline phosphorus & 894.56 & 407.79 & 812.89 & 425.73 & $<0.01$ \\
\hline iron & 14.57 & 10.21 & 13.15 & 8.67 & $<0.01$ \\
\hline sodium & 2.930 & 4.92 & 2.586 & 1.64 & $<0.01$ \\
\hline potassium & 2.794 & 1.54 & 2.610 & 1.52 & $<0.01$ \\
\hline vitamin A & 552.04 & 980.01 & 549.32 & 870.4 & .953 \\
\hline carotene & 3.142 & 5.87 & 3.267 & 5.28 & .651 \\
\hline retinol & 80.72 & 164.66 & 54.62 & 75.57 & $<0.01$ \\
\hline thiamine & 1.50 & 0.71 & 1.41 & 0.72 & $<0.01$ \\
\hline riboflavin & 1.16 & 0.66 & 1.01 & 0.67 & $<0.01$ \\
\hline niacin & 12.27 & 6.39 & 11.07 & 6.72 & $<0.01$ \\
\hline
\end{tabular}


The results in Table 2 show that while vitamin A and carotene showed differences only in mean intake, there were differences in all variables between the two groups. In particular, the Yes-group had lower intake in all nutrients than the No-group, and these differences were statistically significant.

\subsection{Theoretical review: Factors influencing osteoarthritis}

A theoretical review was conducted using binary logistic regression to evaluate the effect of nutrient intake on osteoarthritis depending on the control variables (demographic characteristics). The most significant feature distinguishing logistic regression from traditional regression analysis is that its outcome variable is 0 or 1 . Hence, the linear regression equation, The logistic model expression ensures that the dependent variable or result value is always between ranges $[0,1]$, regardless of which number of the independent variable is $[-\infty, \infty]$. The following explanation describes how the logistic function is obtained:

$$
\operatorname{logit}\left(\mathbb{E} \mid\left[Y_{i} \mid x_{1, i}, \ldots, x_{m, i}\right]\right)=\operatorname{logit}\left(p_{i}\right)=\ln \frac{p_{i}}{1-p_{i}}
$$

Since the outcome of logit transformation in logistic regression is identical to the linear function for $\mathrm{x}$, it becomes

$$
\operatorname{logit}\left(p_{i}\right)=\beta_{0}+\beta_{1} x_{1, i}+\cdots+\beta_{m} x_{m, i}=\beta \cdot X_{i}
$$

When combined lineally it yields (Equation 2),

$$
\ln \frac{p_{i}}{1-p_{i}}=\beta \cdot X_{i}
$$

Therefore, when the explanatory variable $\mathrm{x}$ is given, the probability that the outcome variable belongs to the category becomes the following(Wooldridge J., 2016) (Equation 3),

$$
\mathrm{p}_{i}=\operatorname{logit}^{-1}\left(\beta \cdot X_{i}\right)=\frac{1}{1+e^{-\beta \cdot x_{i}}}
$$

\begin{tabular}{|c|c|c|c|c|c|c|}
\hline \multirow{3}{*}{ Independent Variables } & \multicolumn{3}{|c|}{ Non-Adjusted (Equation 1) } & \multicolumn{3}{|c|}{ Adjusted (Equation 2) } \\
\hline & \multirow{2}{*}{$\begin{array}{l}\text { Odds Ratio } \\
\text { (Std.err) }\end{array}$} & \multicolumn{2}{|c|}{$95 \% \mathrm{CI}$} & \multirow{2}{*}{$\begin{array}{l}\text { Odds Ratio } \\
\text { (Std.err) }\end{array}$} & \multicolumn{2}{|c|}{$95 \% \mathrm{CI}$} \\
\hline & & Min & $\operatorname{Max}$ & & Min & $\operatorname{Max}$ \\
\hline Intercept & $\begin{array}{l}0.85 \\
(0.037)\end{array}$ & & & $\begin{array}{l}0.517 \\
(0.032)\end{array}$ & & \\
\hline proteins & $\begin{array}{l}0.994 \\
(.006)\end{array}$ & .982 & 1.006 & $\begin{array}{l}0.987 \\
(.007)\end{array}$ & .960 & 1.015 \\
\hline fats, & $\begin{array}{l}0.960 \\
(.025)\end{array}$ & .913 & 1.008 & $\begin{array}{l}1.056^{* *} \\
(.026)\end{array}$ & 1.023 & 1.092 \\
\hline saturated fatty-acids & $\begin{array}{l}1.046 \\
(.034)\end{array}$ & .972 & 1.111 & $\begin{array}{l}0.951 \\
(.036)\end{array}$ & .812 & 1.112 \\
\hline monounsaturated fatty-acids & $\begin{array}{l}1.046^{*} \\
(.034)\end{array}$ & .996 & 1.136 & $\begin{array}{l}0.931 * * \\
(.035)\end{array}$ & .801 & .998 \\
\hline polyunsaturated fatty-acids & $\begin{array}{l}1.489 * \\
(.228)\end{array}$ & .952 & 2.329 & $\begin{array}{l}0.726 \\
(.233)\end{array}$ & .157 & 3.344 \\
\hline n-3 fatty-acids & $\begin{array}{l}0.696 \\
(.229)\end{array}$ & .444 & 1.091 & $\begin{array}{l}1.314 \\
(.235)\end{array}$ & .287 & 6.014 \\
\hline n-6 fatty-acids & $\begin{array}{l}0.710 \\
(.220)\end{array}$ & .461 & 1.093 & $\begin{array}{l}1.317 \\
(.225)\end{array}$ & .294 & 5.890 \\
\hline cholesterol & $\begin{array}{l}1.000 \\
(.000)\end{array}$ & 1.000 & 1.001 & $\begin{array}{l}0.999 \\
(.000)\end{array}$ & .997 & 1.001 \\
\hline
\end{tabular}

Using the above equations, an analysis was performed for the effect of nutrient intake on osteoarthritis (Table 3).

Table 3. Odds ratio of osteoarthritis prevalence depending on whether control variables are adjusted. 


\begin{tabular}{|c|c|c|c|c|c|c|}
\hline \multirow{3}{*}{ Independent Variables } & \multicolumn{3}{|c|}{ Non-Adjusted (Equation 1) } & \multicolumn{3}{|c|}{ Adjusted (Equation 2) } \\
\hline & \multirow{2}{*}{$\begin{array}{l}\text { Odds Ratio } \\
\text { (Std.err) }\end{array}$} & \multicolumn{2}{|c|}{$95 \% \mathrm{CI}$} & \multirow{2}{*}{$\begin{array}{l}\text { Odds Ratio } \\
\text { (Std.err) }\end{array}$} & \multicolumn{2}{|c|}{$95 \% \mathrm{CI}$} \\
\hline & & Min & Max & & Min & Max \\
\hline carbohydrate & $1.000(.001)$ & .999 & 1.002 & $1.001(.001)$ & .997 & 1.003 \\
\hline dietary fiber & $0.997(.007)$ & .984 & 1.010 & $0.996(.007)$ & .968 & 1.020 \\
\hline calcium & $0.999 *(.000)$ & .999 & 1.000 & $0.999 * *(.001)$ & .999 & 1.000 \\
\hline phosphorus & $1.000(.000)$ & .999 & 1.001 & $1(.000)$ & .998 & 1.002 \\
\hline iron & $1.013(.010)$ & .994 & 1.032 & 0.951 .010 & .905 & 1.000 \\
\hline sodium & $1.001 * *(.000)$ & 1.000 & 1.002 & $1.001 * * .000$ & 1.000 & 1.002 \\
\hline potassium & $0.999(.000)$ & 1.000 & 1.000 & $1(.000)$ & .999 & 1.000 \\
\hline vitamin A & $1.000(.000)$ & 1.000 & 1.001 & 1.000 .000 & .999 & 1.001 \\
\hline carotene & $0.999(.000)$ & 1.000 & 1.000 & 0.999 .000 & .999 & 1.000 \\
\hline retinol & $0.999 * *(.001)$ & 0.999 & 1.000 & $0.996(.001)$ & .992 & 1.001 \\
\hline thiamine & $0.862(.128)$ & .671 & 1.109 & $.699 * *(.151)$ & .397 & .897 \\
\hline riboflavin & $1.165(.156)$ & .858 & 1.583 & $1.166(.128)$ & .671 & 2.027 \\
\hline niacin & $1.014(.016)$ & .983 & 1.047 & $1.019(.012)$ & .945 & 1.099 \\
\hline
\end{tabular}

a. $\mathrm{p}<0.1^{*}, \mathrm{p}<0.05^{* *}, \mathrm{p}<0.01^{* * *}$.

The following calculations are the estimated osteoarthritis odds ratio across all age groups 50 years old or older. In Equation 1, which did not adjust for control variables in the logistic regression model, greater sodium intake $(\mathrm{OR}=1.001,95 \% \mathrm{CI}[1.000,1.002])$ increased osteoarthritis odds ratio $(0.05 \mathrm{p}$-value significance level). For retinol, $1 \mu \mathrm{g}$ more intake decreased osteoarthritis odds ratio, which was statistically significant. However, the odds ratio calculated from these two nutrients was too low to serve as evidence that they would have a significant effect. In Equation (2), which adjusted for control variables, greater fat intake (OR=1.056, 95\% CI [1.023, 1.092]) and greater sodium intake $(\mathrm{OR}=1.001,95 \% \mathrm{CI}[1.000,1.002])$ increased osteoarthritis odds ratio. By contrast, greater monounsaturated fatty acid intake $(\mathrm{OR}=0.931,95 \% \mathrm{CI}[0.801,0.998])$ and greater vitamin $\mathrm{B} 1$ intake $(\mathrm{OR}=0.699$, $95 \%$ CI [0.397, 0.897]) decreased osteoarthritis odds ratio. These variables were all statistically significant.

Additionally, further analyses of the effect of nutrient intake on osteoarthritis by age was evaluated (Table 4).

Table 4. Odds ratio of osteoarthritis prevalence depending on whether control variables are adjusted including age as a factor

\begin{tabular}{|c|c|c|c|c|c|c|c|c|c|}
\hline \multirow{3}{*}{$\begin{array}{l}\text { Independent } \\
\text { Variables }\end{array}$} & \multicolumn{3}{|c|}{ Age 50-59 } & \multicolumn{3}{|c|}{ Ages 60-69 } & \multicolumn{3}{|c|}{ Age 70 or older } \\
\hline & \multirow{2}{*}{$\begin{array}{l}\text { OR } \\
\text { (Std.err) }\end{array}$} & \multicolumn{2}{|c|}{$95 \% \mathrm{CI}$} & \multirow{2}{*}{$\begin{array}{l}\text { OR } \\
\text { (Std.err) }\end{array}$} & \multicolumn{2}{|c|}{$95 \% \mathrm{CI}$} & \multirow{2}{*}{$\begin{array}{l}\text { OR } \\
\text { (Std.err) }\end{array}$} & \multicolumn{2}{|c|}{$95 \% \mathrm{CI}$} \\
\hline & & Min & $\operatorname{Max}$ & & Min & $\operatorname{Max}$ & & Min & $\operatorname{Max}$ \\
\hline Intercept & $\begin{array}{l}0.69 \\
(0.030)\end{array}$ & & & $\begin{array}{l}0.335 \\
(0.038)\end{array}$ & & & $\begin{array}{l}0.607 \\
(0.030)\end{array}$ & & \\
\hline proteins & $\begin{array}{l}1.001 \\
(.047)\end{array}$ & 0.985 & 1.033 & $\begin{array}{l}1.005 \\
(0.050)\end{array}$ & .983 & 1.027 & $\begin{array}{l}0.988 \\
(0.014)\end{array}$ & .960 & 1.015 \\
\hline fats, & $\begin{array}{l}1.078 \\
(0.057)\end{array}$ & 0.988 & 1.175 & $\begin{array}{l}1.055 \\
(0.053)\end{array}$ & .960 & 1.158 & $\begin{array}{l}1.056 \\
(0.056)\end{array}$ & .950 & 1.173 \\
\hline saturated fatty-acids & $\begin{array}{l}0.958 \\
(0.056)\end{array}$ & 0.851 & 1.078 & $\begin{array}{l}0.959 \\
(0.061)\end{array}$ & .846 & 1.087 & $\begin{array}{l}0.951 \\
(0.076)\end{array}$ & .812 & 1.112 \\
\hline $\begin{array}{l}\text { monounsaturated } \\
\text { fatty-acids }\end{array}$ & $\begin{array}{l}0.891 \\
(0.031)\end{array}$ & 0.786 & 1.008 & $\begin{array}{l}0.946 \\
(0.578)\end{array}$ & .839 & 1.066 & $\begin{array}{l}0.931 \\
(0.070)\end{array}$ & .801 & 1.080 \\
\hline $\begin{array}{l}\text { polyunsaturated } \\
\text { fatty-acids }\end{array}$ & $\begin{array}{l}0.717 \\
(0.314)\end{array}$ & .303 & 1.659 & $\begin{array}{l}0.883 \\
(0.304)\end{array}$ & .449 & 1.734 & $\begin{array}{l}0.726 \\
(0.565)\end{array}$ & .157 & 3.344 \\
\hline
\end{tabular}




\begin{tabular}{|c|c|c|c|c|c|c|c|c|c|}
\hline \multirow{3}{*}{$\begin{array}{l}\text { Independent } \\
\text { Variables }\end{array}$} & \multicolumn{3}{|c|}{ Age 50-59 } & \multicolumn{3}{|c|}{ Ages 60-69 } & \multicolumn{3}{|c|}{ Age 70 or older } \\
\hline & \multirow{2}{*}{$\begin{array}{l}\text { OR } \\
\text { (Std.err) }\end{array}$} & \multicolumn{2}{|c|}{$95 \% \mathrm{CI}$} & \multirow{2}{*}{$\begin{array}{l}\text { OR } \\
\text { (Std.err) }\end{array}$} & \multicolumn{2}{|c|}{$95 \% \mathrm{CI}$} & \multirow{2}{*}{$\begin{array}{l}\text { OR } \\
\text { (Std.err) }\end{array}$} & \multicolumn{2}{|c|}{$95 \% \mathrm{CI}$} \\
\hline & & Min & Max & & Min & $\operatorname{Max}$ & & Min & Max \\
\hline n-3 fatty-acids & $\begin{array}{l}1.377 \\
(0.611)\end{array}$ & .576 & 3.288 & $\begin{array}{l}1.006 \\
(0.352)\end{array}$ & .506 & 2.000 & $\begin{array}{l}1.314 \\
(1.020)\end{array}$ & .287 & 6.014 \\
\hline n-6 fatty-acids & $\begin{array}{l}1.261 \\
(0.546)\end{array}$ & .539 & 2.950 & $\begin{array}{l}1.052 \\
(0.341)\end{array}$ & .557 & 1.969 & $\begin{array}{l}1.318 \\
(1.010)\end{array}$ & .294 & 5.890 \\
\hline cholesterol & $\begin{array}{l}1.01 * * \\
(0.001)\end{array}$ & 1.000 & 1.002 & $\begin{array}{l}0.999 \\
(0.001)\end{array}$ & .998 & 1.001 & $\begin{array}{l}0.999 \\
(0.001)\end{array}$ & .997 & 1.001 \\
\hline carbohydrate & $\begin{array}{l}1.001 \\
(0.001)\end{array}$ & .993 & .999 & $\begin{array}{l}0.998 \\
(0.001)\end{array}$ & .996 & 1.001 & $\begin{array}{l}1 \\
(0.001)\end{array}$ & .997 & 1.003 \\
\hline dietary fiber & $\begin{array}{l}1.008 \\
(0.013)\end{array}$ & .980 & 1.035 & $\begin{array}{l}1.012 \\
(0.011)\end{array}$ & .989 & 1.036 & $\begin{array}{l}0.996 \\
(0.013)\end{array}$ & .968 & 1.023 \\
\hline calcium & $\begin{array}{l}0.978 * * \\
(0.001)\end{array}$ & .965 & .998 & $\begin{array}{l}1 \\
(0.001)\end{array}$ & .998 & 1.001 & $\begin{array}{l}1.001 \\
(0.001)\end{array}$ & .999 & 1.002 \\
\hline phosphorus & $\begin{array}{l}0.999 \\
(0.001)\end{array}$ & .997 & 1.001 & $\begin{array}{l}0.999 \\
(0.001)\end{array}$ & .997 & 1.001 & $\begin{array}{l}1 \\
(0.001)\end{array}$ & .998 & 1.002 \\
\hline iron & $\begin{array}{l}0.989 \\
(0.023) \\
\end{array}$ & .945 & 1.035 & $\begin{array}{l}0.995 \\
(0.011)\end{array}$ & .974 & 1.016 & $\begin{array}{l}0.952 * * \\
(0.024)\end{array}$ & .905 & 0.997 \\
\hline sodium & $\begin{array}{l}1.001 * * \\
(0.001)\end{array}$ & 1.000 & 1.002 & $\begin{array}{l}0.969 \\
(0.001)\end{array}$ & .919 & 1.000 & $\begin{array}{l}1 \\
(0.001)\end{array}$ & .999 & 1.001 \\
\hline potassium & $\begin{array}{l}0.999 * * \\
(0.001)\end{array}$ & 0.999 & 0.999 & $\begin{array}{l}0.999 \\
(0.001)\end{array}$ & .999 & 1.000 & $\begin{array}{l}1 \\
(0.001)\end{array}$ & .999 & 1.000 \\
\hline vitamin A & $\begin{array}{l}0.999 \\
(0.001) \\
\end{array}$ & .997 & .999 & $\begin{array}{l}0.999 \\
(0.001) \\
\end{array}$ & .998 & 1.001 & $\begin{array}{l}1 \\
(0.001) \\
\end{array}$ & .998 & 1.002 \\
\hline carotene & $\begin{array}{l}1.001 \\
(0.001)\end{array}$ & .998 & 1.000 & $\begin{array}{l}1 \\
(0.001)\end{array}$ & .999 & 1.000 & $\begin{array}{l}1 \\
(0.001)\end{array}$ & .999 & 1.001 \\
\hline retinol & $\begin{array}{l}0.999 \\
(0.001)\end{array}$ & .996 & 1.002 & $\begin{array}{l}0.998 \\
(0.002)\end{array}$ & .994 & 1.001 & $\begin{array}{l}1 \\
(0.002)\end{array}$ & .992 & 1.001 \\
\hline thiamine & $\begin{array}{l}1.232 \\
(0.352)\end{array}$ & .703 & 2.158 & $\begin{array}{l}0.83 * * \\
(0.301)\end{array}$ & .790 & .892 & $\begin{array}{l}0.699 \\
(0.201)\end{array}$ & .397 & 1.234 \\
\hline riboflavin & $\begin{array}{l}0.687 \\
(0.229)\end{array}$ & .356 & 1.323 & $\begin{array}{l}0.981 * \\
(0.23)\end{array}$ & .482 & 1.429 & $\begin{array}{l}1.166 \\
(0.329)\end{array}$ & .671 & 2.207 \\
\hline niacin & $\begin{array}{l}1.001 \\
0.012\end{array}$ & .944 & 1.062 & $\begin{array}{l}1.01 \\
(0.011)\end{array}$ & .957 & 1.067 & $\begin{array}{l}1.019 \\
(0.039)\end{array}$ & .945 & 1.099 \\
\hline
\end{tabular}

a. $\mathrm{p}<0.1^{*}, \mathrm{p}<0.05^{* *}, \mathrm{p}<0.01^{* * *}$.

b. Control variables: Income, place of residence, education level, type of occupation, marital status, private health insurance, economic activity, smoking, and drinking.

c. $n=2,084$.

The results showed that the osteoarthritis odds of women 50-59 years old was higher when cholesterol intake was higher $(\mathrm{OR}=1.010,95 \% \mathrm{CI}[1.000,1.002])$ and when sodium intake was higher $(\mathrm{OR}=1.001,95 \% \mathrm{CI}[1.000$, 1.002]). Greater calcium intake $(\mathrm{OR}=0.978,95 \% \mathrm{CI}[0.965,0.998])$ and greater potassium intake $(\mathrm{OR}=0.999,95 \%$ CI $[0.999,0.999])$ tended to decrease osteoarthritis prevalence with statistical significance.

For women 60-69 years old, greater vitamin $\mathrm{B} 1$ intake $(\mathrm{OR}=0.830,95 \% \mathrm{CI}[0.790,0.892])$ and greater vitamin $\mathrm{B} 2$ intake (OR $=0.981,95 \% \mathrm{CI}[0.482,1.429])$ reduced osteoarthritis odds. Nevertheless, the variable that was found significant within the present study's significance level (p-value 0.05) was vitamin B1, and vitamin B2 was statistically significant at $10 \%$.

For women 70 years old or older, more iron intake $(\mathrm{OR}=0.952,95 \%$ CI $[0.905,0.997])$ decreased osteoarthritis 
odds with statistical significance.

\section{Discussion}

Summarizing the results of this study, among women 50-59 years old, cholesterol and sodium increased osteoarthritis prevalence, while nutrients classified as minerals reduced osteoarthritis prevalence. Further, B vitamins decreased osteoarthritis prevalence in women 60-69 years old, and iron was found to have a significant effect on women 70 years old or older.

A study by the Ministry of Food and Drug Safety (MFDS, formerly known as the Korea Food \& Drug Administration or KFDA) reported that minerals are the nutrients that primarily form bones and influence bone metabolic homeostasis(KFDA., 2012), and the results of the current study are consistent with the MFDS study's conclusions. Because B vitamins alleviate edema, help the blood to circulate better, and protect nerves, B vitamins help relieve arthritis(Lee, 2014). Furthermore, another study on the osteoarthritis prevalence of people 65 years old or older in South Korea also confirmed that B vitamins and riboflavin had a significant effect on osteoarthritis prevalence. The present study's results are consistent with those from earlier studies.

These results seem to be attributable to a lack of the intake of the so-called 'micronutrients' (such as minerals and vitamins) among women 50 years old or older compared to men and younger women(Koo et al., 2014; Choi et al, 2007). These nutrients are most important for bone health, and their low levels among middle-aged or post-menopause women seem to have contributed to the present study's results. As bone mass itself is not determined at a particular point in time but slowly changes throughout a person's life(Choi et al., 2007), it would be necessary to conduct a longitudinal study, which explores ways to manage bone mass across various age groups.

The study analyzed the effect of nutrient intake on osteoarthritis based on data from the 7th KNHANES in 2016 and 2017. The results showed that nutrients influenced osteoarthritis prevalence differently across age groups. Minerals, vitamins, and iron were found to have a statistically significant effect on osteoarthritis in women 50-59 years old, 60-69 years old, and 70 years old or older, respectively. These nutrients are 'micronutrients' and, among the five major nutrients, were identified as the nutrients that assisted the 'macronutrients' (carbohydrates, fats, and proteins).

Due to its limitation as a cross-sectional study that could only show whether there was a correlation with osteoarthritis, the present study could not examine each correlation over time, nor could it use data based on a 24-hour dietary recall method to reflect participants' daily average intake. However, the present study is critical because it used data from KNHANES, which sampled all people in Korea, and identified more representative and accurate factors influencing osteoarthritis.

Based on these results, further research is recommended. It is necessary to conduct a study that analyzes and compares the correlation between nutrient intake and osteoarthritis prevalence across different editions of KNHANES data. Further, a study that provides nutrition education programs to osteoarthritis patients and evaluates their osteoarthritis-related pain and quality of life is needed(Kim., 2016). Finally, it is crucial to develop a protocol for educating osteoarthritis patients about nutritional needs at different ages.

\section{Competing Interests Statement}

The authors declare that there are no competing or potential conflicts of interest.

\section{References}

Asan medical center. (2019). Arthritis. $\quad$ Retrieved from http://www.amc.seoul.kr/asan/healthinfo/disease/diseaseDetail.do? contentId=31713.

Breedveld, F. C. (2004). Osteoarthritis--the impact of a serious disease. Rheumatology, 43(1), 4-8. https://doi.org/10.1093/rheumatology/keh102

Centers for disease control \& prevention. (2019). Osteoarthritis. Retrieved from http://www.cdc.go.kr/CDC/cms/content/mobile/19/70119_view.html.

Cho, H. J., Chang, C. B., \& Kim, K. W. (2011). Gender and prevalence of knee osteoarthritis types in elderly Koreans. The Journal of Arthroplasty, 26(7), 994-999. https://doi.org/10.1016/j.arth.2011.01.007

Choi, W. S., Lee, G., Song, W. H., Koh, J. T., Yang, J., Kwak, J. S., ..., \& Chun, J. S. (2019). The CH25H-CYP7B1-ROR $\alpha$ axis of cholesterol metabolism regulates osteoarthritis. Nature, 566, 254-258. https://doi.org/10.1038/s41586-019-0920-1

Choi, M. K., \& Kim, M. H. (2007). A study on bone mineral density, dietary habits and nutritional status of adult women in the three age groups. Journal of the Korean Society of Food Culture, 22(6), 833-840. 
Fauci, A., Kasper, D., Longo, D., Braunwald, E., Hauserm, S., \& Jameson, J. (2008). Harrison's internal principles of internal medicine (17th ed.). McGraw-Hill Medical, New York,

Gardiner, B. S., Woodhouse, F. G., \& Besier, T. F. (2015). Predicting knee osteoarthritis. Annals of Biomedical Engineering, 44, 222-233. https://doi.org/10.1007/s10439-015-1393-5

Ha, W. K., Park, K. K., Kim, T. H., Lee, K. H., Lee, Y. J., \& Chung, W. J. (2019). Association between arthritis and socio-demographic factors in Korean elderlies: The national survey of Korean elderly $(2014,2017)$ Dataset Analysis. Health Policy and Management, 29(4), 469-481.

Hur, N. W., Choi, C. B., Uhm, W. S., \& Bae, S. C. (2008). The prevalence and trend of arthritis in Korea: Results from Korea national health and nutrition examination surveys. The journal of the Korean Rheumatism Association, 15, 11-16. https://doi.org/10.4078/jkra.2008.15.1.11

Kim, S. Y., \& Jeon, E. Y. (2011). Influencing factors on osteoarthritis in Korean. The Korean Journal of Rehabilitation Nursing, 14(2), 111-117.

Kim, Y. B. (2016). Strategies to approach the customized health management service to prevent chronic diseases. Korean Society For Health Education And Promotion, 33(4), 89-100. https://doi.org/10.14367/kjhep.2016.33.4.89

KFDA. (2012). Health function food functionality assessment guide - 'Helping Bone/joint Health'.

Koo, J. O., \& Kim, M. S. (2014). Analysis of Bone Mineral Density, Biochemical index and nutrient intakes of 30-70 years old women - based on 2011 KNHANES. Korean Journal of Community Nutrition, 19(4), 328-341. https://doi.org/10.5720/kjen.2014.19.4.328

Lee, H. S. (2014). Prevalence of osteoarthritis and related risk factors in the elderly: Data from the Fifth Korea national health and nutrition examination survey (KNHANES V). 2010-2012. Journal of the Korean Dietetic Association, 20(2), 99-109. https://doi.org/10.14373/JKDA.2014.20.2.99

Pearson-Ceol, J. (2007). Literature review on the effects of obesity on knee osteoarthritis. Orthopaedic Nursing, 26(5), 289-292. https://doi.org/10.1097/01.NOR.0000295955.63956.1d

Spector, T. D., Cicuttini, F., Baker, J., Loughlin, J., \& Hart, D. (1996). Genetic influences on osteoarthritis in women: a twin study. Bmj, 312, 940-943. https://doi.org/10.1136/bmj.312.7036.940

Wooldridge, J. (2016). Introductory econometrics: A modern approach (6th ed.). CENGAGE Learning. Boston.

Zakkak, J. M., Wilson, D. B., \& Lanier, J. O. (2009). The association between body mass index and arthritis among US adults: CDC's surveillance case definition. Preventing Chronic Dissease, 6(2), A56. PMID: 19288999

\section{Copyrights}

Copyright for this article is retained by the author(s), with first publication rights granted to the journal.

This is an open-access article distributed under the terms and conditions of the Creative Commons Attribution license (http://creativecommons.org/licenses/by/4.0/). 